
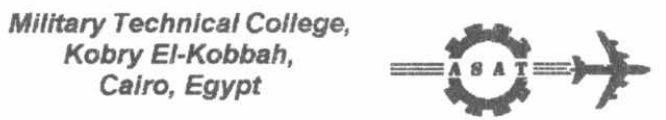

$9^{\text {th }}$ International Conference

On Aerospace Sciences \&

Aviation Technology

\title{
VORTEX SIZE CONTROL FOR ORIFICES CASCADE IN A RECTANGULAR DUCT
}

\author{
LOTFY*A. H., SALEH* I., ABDALLAH*H. M., SALAH* A. M.
}

\begin{abstract}
Vortex formation and shedding downstream obstructions may be assumed to be one of the main sources of flow induced vibration and noise in pipes, ducts, and control valves. The level of the produced noise depends on the size of the formed vortex and its shedding frequency hence, controlling the size of the formed vortex may be one of the main factors that help in attenuation of the generated noise. The vortex size may be estimated from the reattachment length of the separated flow downstream these obstructions. In the present work, vortex formation downstream a single orifice and two orifices in series in a rectangular duct have been investigated numerically and experimentally. A numerical solution has been coded to solve the flow governing equations in the primitive variables using the finite difference technique. The solution has been carried out for laminar, 2-D and incompressible flow field at Reynolds number ranges from 50 to 400 for orifice height to duct height ratio of 0.5 and for the inter distance between the two orifices from $0.2 \mathrm{H}$ to $7 \mathrm{H}$. The velocity field, the streamlines, and the vorticity field have been determined. The code is written in visual $\mathrm{C}$-language to achieve low computational time and lower number of iterations. An experimental investigation has been carried out on 2-D laminar flow visualization table. The streamlines of water flow throughout the single orifice and two orifices in the rectangular duct have been visualized for various Reynolds number and orifice height to duct height ratio, $\mathrm{D} / \mathrm{H}$, values. The experimental and the theoretical results showed a considerable agreement. The results showed a minimum vortex size as well as lowest circulation in case of using an inter distance of about $(1-D / H)$. Finally, the results showed that the control of the inter distance between the two orifices or similarly between any two obstructions in a rectangular duct may be of special interest to reduce the size of the generated vortex and consequently to attenuate the vibration and noise.
\end{abstract}

\section{KEY WORDS}

Reattachment length, Circulation, Vortex, Laminar, Noise, streamlines.

*Egyptian Armed Forces 


\section{NOMENCLATURE}

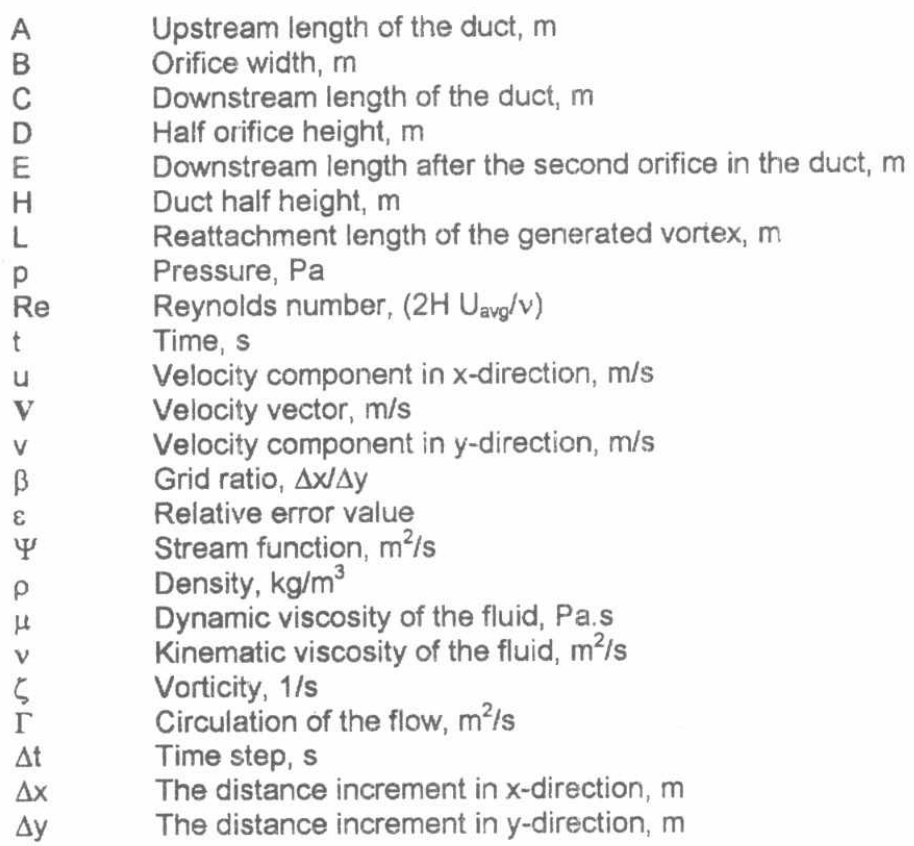

\section{Introduction:}

Pipe network is used to convey fluids to hydraulic and pneumatic actuators in many engineering applications. In fluid power systems, pipes and valves are the main target of study from the point of view of noise and vibration control.

Attenuation of vibration in fluid structure interaction becornes nowadays an important topic for research because of improper and unpleasant effects of the vibration and noise. The structure may loose its expected performance and reaches the ultimate strength of its material when it is subjected to large vibration amplitudes or when the vibration frequency coincides with the structural nature frequency. The fluid structure interaction may be external, as flow of water around bridge foots, or may be internal, as flow in ducts. These types lead to form vortices in the near wake region in case of external flows or after the restriction zones in case of internal flows.

Vortex formation and shedding mechanisms are of great importance due to its nature in acting as a source of vibration. These vortices should take considerable attention in design process, and its attenuation should be controlled either actively or passively.

The generated vortex may be controlled either by modulating its amplitude or its shedding frequency. The vortex reattachment length may be assumed for the vortex size as well as the circulation a measure for the vortex amplitude. 
Mechanisms of vortex formation and shedding due to fluid body interaction are presented by Donald Rockwell, [1]. He explained and analyzed in details how the vortex is formed and shed as well as the affecting parameters on these mechanisms.

A numerical analysis of laminar flow through square-edged orifice had been studied by Sahin B., [2], for Reynolds number in the range $0<R e<2000$ with beta values varying from 0.2 to 0.8 and with $t^{*}$ values varying from $1 / 16$ to 1 . It was shown that the flow discharge coefficient gradually decreased when the orifice thickness/diameter ratio increased for a high porosity.

Tienfuan Kerh, [3], investigated numerically the interaction of a viscous incompressible fluid with a control valve which was conducted by using the finite element method and the network approach. Variables including displacement, velocity and acceleration were presented as a function of time to describe the movements of the solid system. The velocity vector, pressure variations, pressure loss coefficient, and energy losses were displayed to reveai the resulting flow fields. It was observed that the motion of the structure element, which became a moving boundary, had significant influences on the flow fields.

Ahmed F. Ghonem, [4], studied the unsteady flow over a rearward-facing step at a wide range of $\mathrm{Re}$ number. The simulation was restricted to two-dimensional flow. Attention was focused on predicting the effect of Re number on the dynamic structure of flow field in terms of process of vortex eddy formation and interaction. The use of vortex element to represent the vorticity field and its dynamics in a numerical simulation was a natural way to overcome the nonlinearity of Navier-Stokes equations without excessive numerical diffusion. The conclusion declared that the structure of the recirculation zone formed behind the rearward facing step in the channel at low Re number forming an stationary eddy at the corner of the step. When the $\mathrm{Re}$ was introduced, the eddy was dettached and moved towards the downstream direction while the circulating zone increased.

Badekas, [5], studied numerically and compared with a previous experimental work, the solution of laminar axisymmetric flow throughout a sudden expansion in a pipe for $50<\operatorname{Re}<500$ and $1.5<\mathrm{D} / \mathrm{d}<6$ by applying simple algorithm based upon the primitive variables. The accuracy of the numerical solution was established by refinement study and comparison with the previous results. The results gave a flow field for $50<\operatorname{Re}<500$ and $D / d=6$. The results showed also that the reattachment length was observed to be a linear function with $R e$ and $D / d$.

Chang, [6], investigated a modified Reynolds number with $k-\varepsilon$ turbulence model. The performance of the proposed model was assessed through testing with fully developed pipe flows and recirculating flow in pipe expansion. Attention was specifically focused on the flow regime near the point of reattachment. It was shown that the proposed model was capable of correctly predicting the near wall limiting flow behavior while avoiding occurrence of the singular difficulty near the reattaching point as applied to the recirculating flow in sudden expansion pipe.

Robert, [7]. Studied a numerical investigation for the behavior of the vortical wake created by a square cylinder placed in laminar boundary layer flow. Solving the 2-D unsteady equations with a finite volume method performs the calculations. The 
Reynolds number regime investigated was from 500 to 1500 . Another parameter that was varied was the distance of the cylinder from the wall. The initial and the subsequent development of vortex shedding mechanism that investigated in the presence of the wall were found to have strong effects on the properties of these vortices, as well as on the lift, the drag and Strouhal number.

\section{Basic Equations:}

The conservation equations for incompressible fluid flow in horizontal direction, with constant properties and without external heat addition, could be written as :

Continuity equation:

$$
\vec{\nabla} \bullet \vec{V}=0
$$

Momentum equation:

$$
\rho \frac{\mathrm{D} \overrightarrow{\mathrm{V}}}{\mathrm{Dt}}=-\vec{\nabla} \mathrm{p}+\mu \nabla^{2} \overrightarrow{\mathrm{V}}
$$

These equations represent a mixed set of elliptic-parabolic equations contain the unknowns $(V, p)$. For $2-D$ incompressible flow, these equations may be written in a cartesian coordinates as :

Continuity equation:

$$
\frac{\partial u}{\partial x}+\frac{\partial v}{\partial y}=0
$$

$x$-momentum equation:

$$
\frac{\partial u}{\partial t}+u \frac{\partial u}{\partial x}+v \frac{\partial u}{\partial y}=\frac{-1}{\rho} \frac{\partial p}{\partial x}+v\left(\frac{\partial^{2} u}{\partial x^{2}}+\frac{\partial^{2} u}{\partial y^{2}}\right)
$$

$y$-momentum equation

$$
\frac{\partial v}{\partial t}+u \frac{\partial v}{\partial x}+v \frac{\partial v}{\partial y}=\frac{-1}{\rho} \frac{\partial p}{\partial y}+v\left(\frac{\partial^{2} y}{\partial x^{2}}+\frac{\partial^{2} v}{\partial y^{2}}\right)
$$

These equations are written in what so-called the primitive variables form, where the velocity components in $\mathrm{x}$ and $\mathrm{y}$ directions are $\mathrm{u}$ and $\mathrm{v}$, respectively.

\section{Stream Function and Vorticity Transport Equations:}

Although it is possible to obtain numerical solutions from the primitive variable equations, most successful numerical solutions for 2-D flow have utilized the vorticitystream function approach by replacing the primitive variables with both the vorticity $\xi$ and stream function $\psi$.

The vorticity-stream function approach is one of the most popular methods for solving the 2-D incompressible Navier-Stokes equations.

The stream function $\psi$ is defined by:

$$
\mathrm{u}=\frac{\partial \psi}{\partial \mathrm{y}} \quad \mathrm{v}=-\frac{\partial \psi}{\partial \mathrm{x}}
$$


The vorticity vector is defined by

$$
\vec{\xi}=\nabla \times \vec{V}
$$

For 2-D flow, in Cartesian coordinate, the vorticity in z-direction is expressed as:

$$
\xi=\frac{\partial v}{\partial x}-\frac{\partial u}{\partial y}
$$

Eq.(2) and Eq.(3) could be simplified by eliminating the pressure term by differentiate Eq.(2) with respect to $y$ and Eq.(3) with respect to $x$, and substituting by Eq.(6) then, the parabolic vorticity transport equation could be expressed in vector form as:

$$
\frac{\partial \vec{\xi}}{\partial \mathrm{t}}=-\mathrm{V} \cdot(\nabla \vec{\xi})+v^{2} \vec{\xi}
$$

Since the present flow problem is a 2-D problem, the vorticity stream function approach will be used for the following reasons:

1. Reduced number of equations (two equations instead of three equations).

2. It is possible to separate the 2-D, incompressible mixed elliptic Navier-Stokes equations into one parabolic equation (Vorticity-transport equation) and one elliptic equation (Poisson's equation).

The Vorticity transport equation is a parabolic partial differential equation; non-linear as $u$ and $v$ are functions of the dependent variable $\xi$. The equation is parabolic in time, i.e., it possesses a marching or initial value problem, where the solution is stepped out from an assumed initial condition.

The Poisson's equation is an elliptic partial differential equation, i.e., it possesses a boundary value problem, which could be solved by iterative methods.

These two equations have been solved numerically by using the finite difference technique. Herein, the time dependent behavior is not required. Only, the steady state solution is required, which is obtained from the asymptotic value of the time dependent solution of the equation.

\section{Finite Difference Scheme:}

The transformation of the main equations to the finite difference scheme form is presented in [8] which give a complete explanation of these differencing steps. Dynamic instability of the solution is characterized by an oscillatory overshoot with increasing amplitude. Such instability could be rectified by using a smaller $\Delta t$ for the vorticity transport equation. A linear analysis suggests a critical time step above which the dynamic instability will be anticipated. Roache, [9], introduced the following criterion

$$
\Delta t_{c t} \leq \frac{1}{(2 v)\left(\frac{1}{\Delta x^{2}}+\frac{1}{\Delta y^{2}}\right)+\frac{\left|u_{i, j}\right|_{\max }}{\Delta x}+\frac{\left|v_{i, j}\right|_{\max }}{\Delta y}}
$$


To insure stability, only a fraction of this critical time step is used, usually 85 to $95 \%$ of the critical time step $\Delta t$ (critical), according to Roache, [9], and Wirz, [10]. In the present work, a value of $95 \%$ is used.

At every grid point, the error, $\varepsilon$, has been calculated as follows:

$$
\varepsilon=\left|X_{i, j}^{k+1}-X_{i, j}^{k}\right|
$$

Where $X$ stands for $\zeta$ or $\psi$. Taking the maximum values across the whole domain, this value must not exceed the required degree of accuracy. If this value were not achieved, another iterations carried out until the required value is achieved. Stream function $\psi$ is permitted to use only finite number of five iterations not to reach a certain error, which gives satisfactory accuracy, and a limited number of iterations. At the end, the solution should satisfy the error for both $\psi$ and $\zeta$. In the present work the value of error for the stream function is chosen to be $10^{-6}$ and for the vorticity to be $10^{-6}$.

\section{Boundary Conditions for Vorticity Stream Function:}

The present case of study is a boundary value problem. Therefore, it is important to specify the boundary conditions, with giving a special care to the corners. The boundary condition for the single orifice is the same as that stated for the two orifices in series for each wall and inlet/outlet conditions. These boundary conditions are explained in details in [8] and [12].

The boundary conditions for the studied domain, as shown in Fig. 1, are used for the velocity, stream function and vorticity as follows:

For the inlet conditions, far from the orifice, it is suggested that the flow has a uniform profile.

For the outlet conditions, far enough from the orifice, it is suggested that the flow back again has a uniform profile.

For the wall conditions, from no slip conditions, the flow velocity has a zero value and it is suggested as for the stream function.

For the corners, it is treated as a singularity point.

\section{Results:}

The results of the theoretical model have been carried out for the following conditions and limitations.

\section{Input Data:}

The solution has been carried out for the following parameters:

The input data are: $\mathrm{dx}=0.2 \mathrm{~cm}, \mathrm{dy}=0.5 \mathrm{~cm}$ : $\mathrm{dt}=0.95 \Delta \mathrm{tcr}$

Duct dimension for the upstream and downstream sections are selected according to the Fluid Handbook, [11], related to Fig.1 and Fig.2:
Duct half height
$\mathrm{H}=20 \mathrm{~cm}$
Upstream length
$A=H(20 \mathrm{~cm})$
Orifice width
$B=0.1 \mathrm{H}(1 \mathrm{~cm})$ 


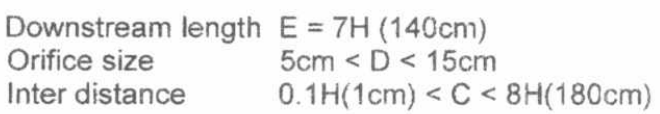

The code is written by visual $\mathrm{C}++$ language, the total time needed to solve the flow domain is 20 minutes, the size of the source program is $12.6 \mathrm{MB}$, the size of the executable file is $35 \mathrm{kB}$.

The solution was converged after a suitable number of iterations, which indicated the stability of the solution; the error for the stream function is strongly converged without any oscillations, which is the advantage of using the stream function-vorticity approach.

These contours represent the variation of the stream function and the vorticity values at different cases of study for the domain under investigations.

Fig. 3 and Fig. 4 represent contour plots for the stream function and the vorticity for a single orifice at the ratio $\mathrm{D} / \mathrm{H}=0.5, \mathrm{Re}=200$ and 300 .

Fig. 5 and Fig. 6 represent contour plots for the stream function for two orifices in series at the ratio $\mathrm{D} / \mathrm{H}=0.5, \mathrm{Re}=100$ and inter distance between the two orifices $3 \mathrm{H}$ and $\mathrm{H}$

\subsection{Reattachment Length}

Fig. 7, Fig. 9 and Fig. 11 show the variation of the reattachment length downstream the second orifice at different $\mathrm{Re}$ and different inter distance between the two orifices at $\mathrm{D} / \mathrm{H}=0.5$.

The results showed that the reattachment length downstrearn the second orifice has an optimum length (minimum vortex size) corresponding to the value of $(1-\mathrm{D} / \mathrm{H})$ of the duct height, which could be considered as a square cavity of equal sides where the flow has a minimum vortex size.

\subsection{Circulation}

The circulation downstream the second orifice has been calculated for different $R e$ and at different geometry parameters (D/H). Fig. 8, Fig. 10 and Fig. 12 show the variation of the circulation downstream the second orifice at different $\mathrm{Re}$ and at different inter-distance between the two orifices at $\mathrm{D} / \mathrm{H}=0.5$.

The results showed that the circulation downstream the second orifice has an optimum (minimum vortex size) corresponding to the value of $(1-\mathrm{D} / \mathrm{H})$ of the duct height. 


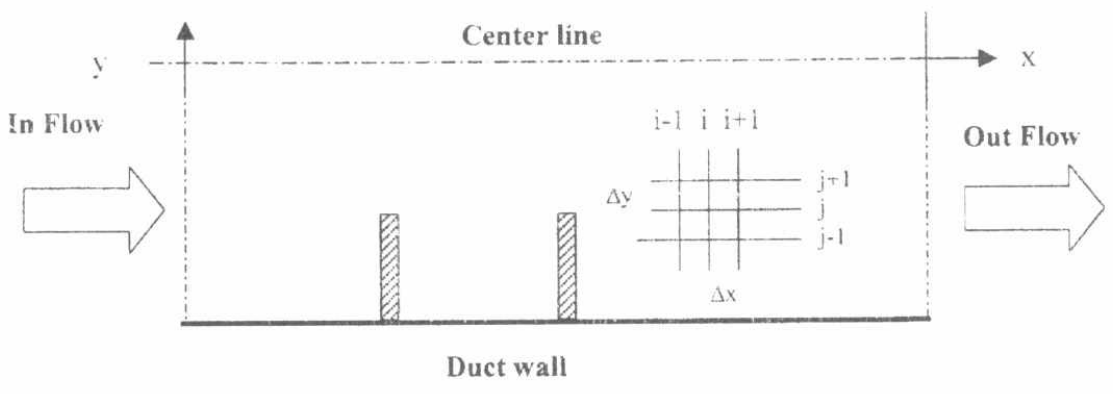

Fig. 1. Scheme of the Duct

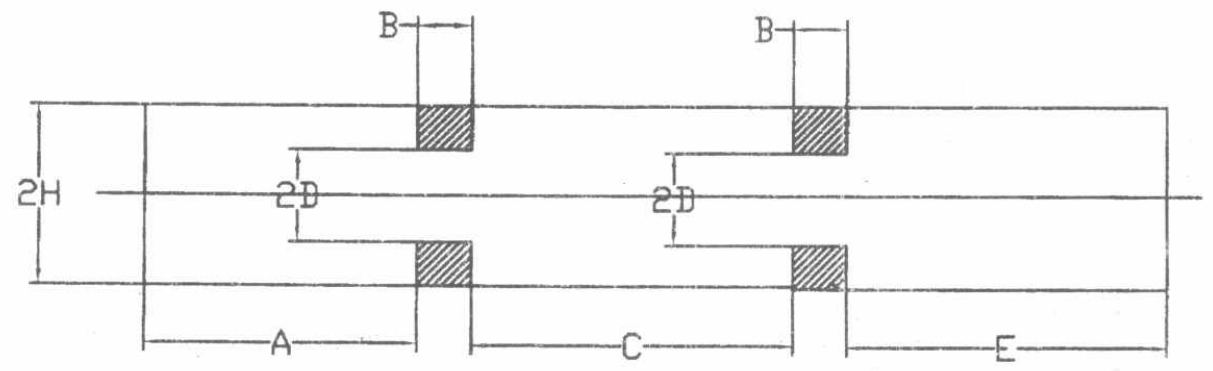

Fig.2. Duct and Orifice Dimensions 


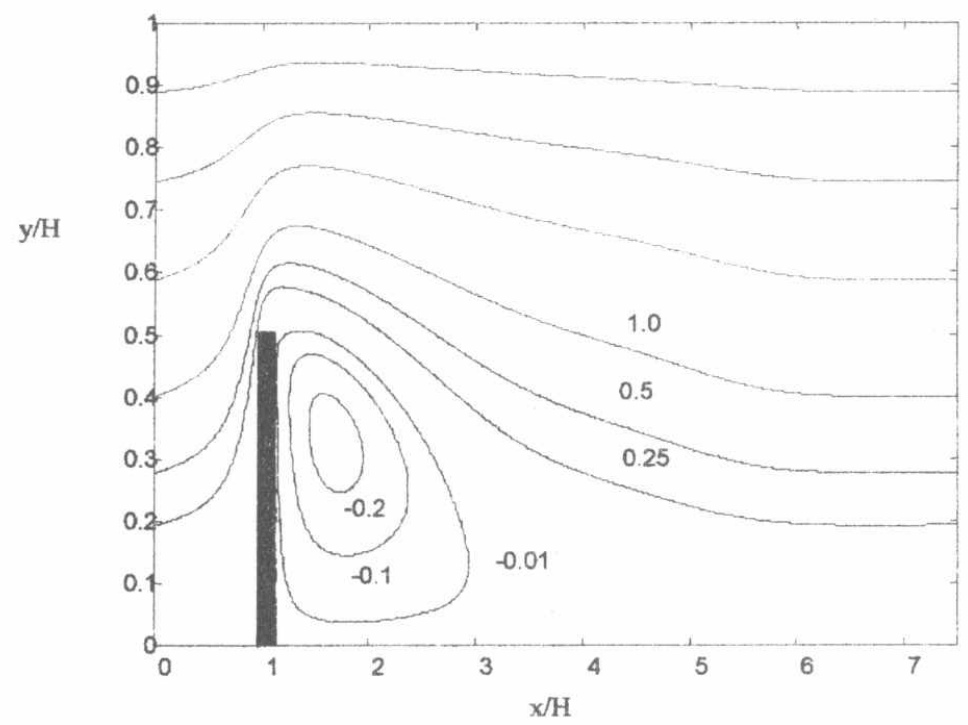

Fig. 3 Stream function at $\mathrm{Re}=200$ and $\mathrm{D} / \mathrm{H}=0.5$ $(\Delta x=0.2, \Delta y=0.5$ and total grid points 30000$)$

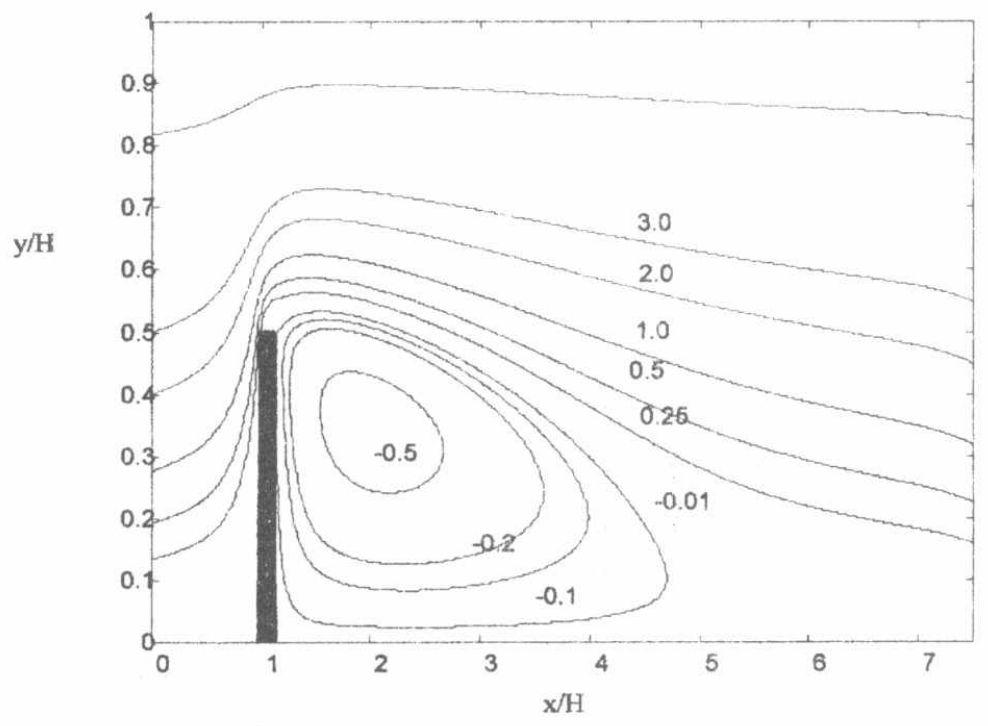

Fig. 4 Stream function at $\mathrm{Re}=300$ and $\mathrm{D} / \mathrm{H}=0.5$ $(\Delta x=0.2, \Delta y=0.5$ and total grid points 30000$)$ 


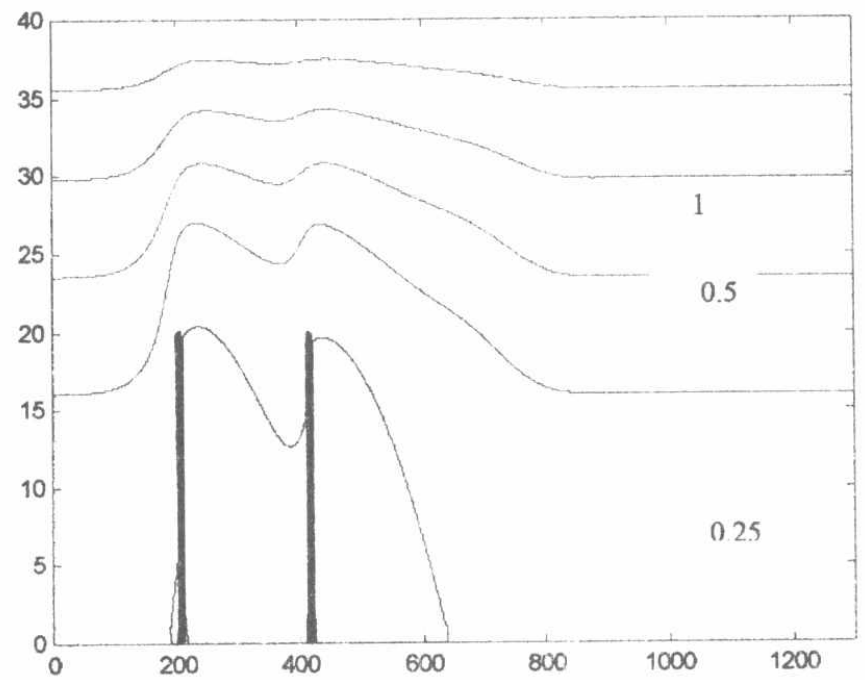

Fig. 5 Two orifices in series Stream Lines Values, $\mathrm{cm}^{2} / \mathrm{s}$ $(\mathrm{Re}=100, \mathrm{D} / \mathrm{H}=0.5$, Inter Distance $=3 \mathrm{H})$

j

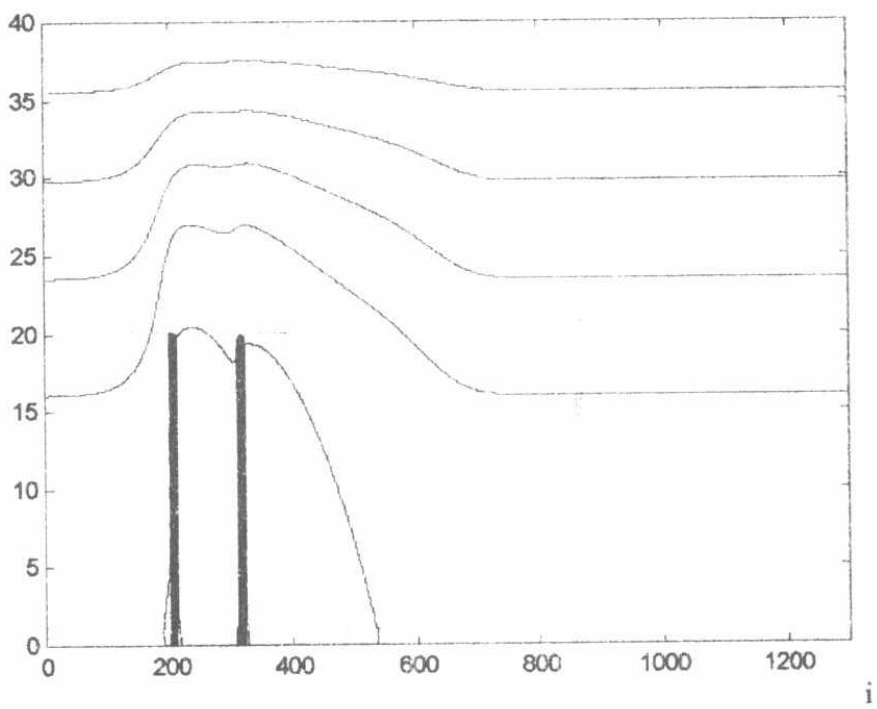

Fig. 6 Two orifices in series Stream Lines Values, $\mathrm{cm}^{2} / \mathrm{s}$ $(\operatorname{Re}=100, \mathrm{D} / \mathrm{H}=0.5$, Inter Distance $=\mathrm{H})$ 


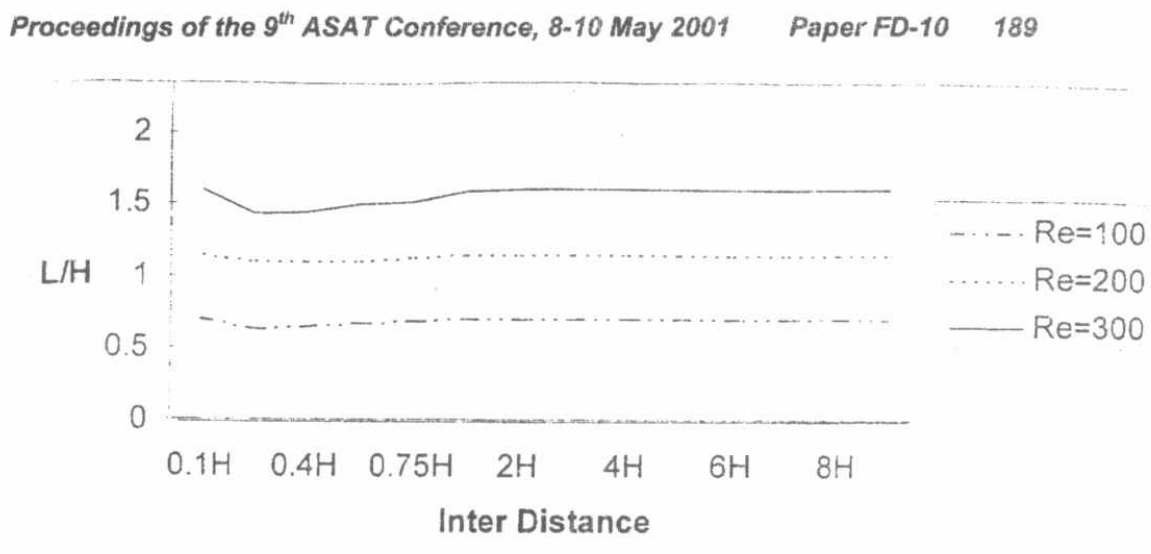

Fig. 7 The Behavior of the Reattachment length to the Duct height ratio at different inter distances between the two orifices

(Results for $\mathrm{D} / \mathrm{H}=0.7$ )

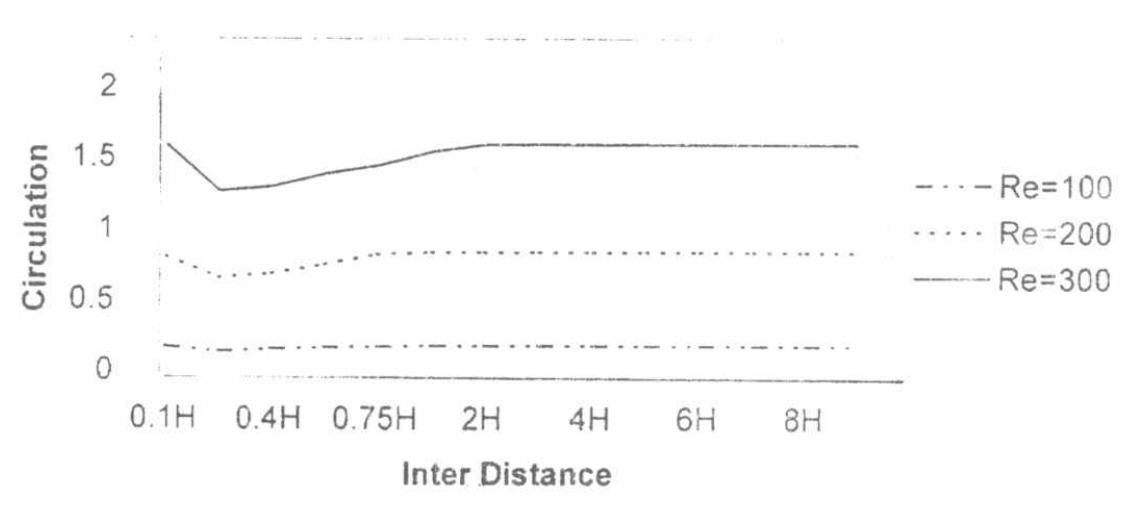

Fig. 8 The Behavior of the circulation at different inter distances between the two orifices

(Results for $\mathrm{D} / \mathrm{H}=0.7$ ) 


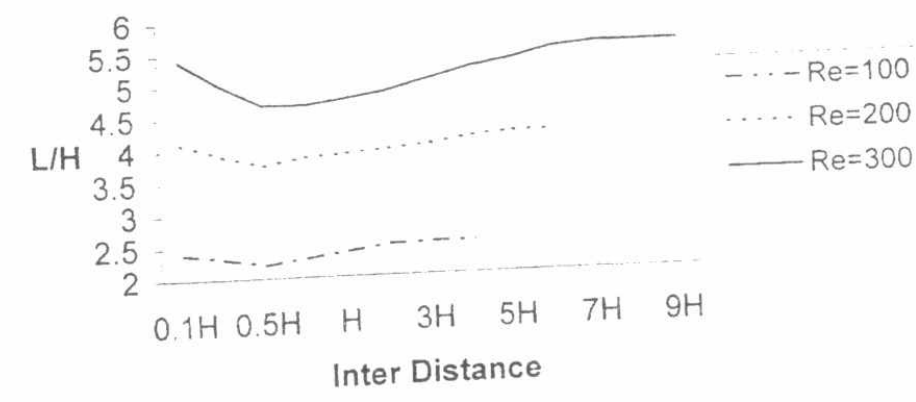

Fig. 9 The Behavior of the Reattachment length to the Duct height ratio at different inter distances between the two orifices (Results for $\mathrm{D} / \mathrm{H}=0.5$ )

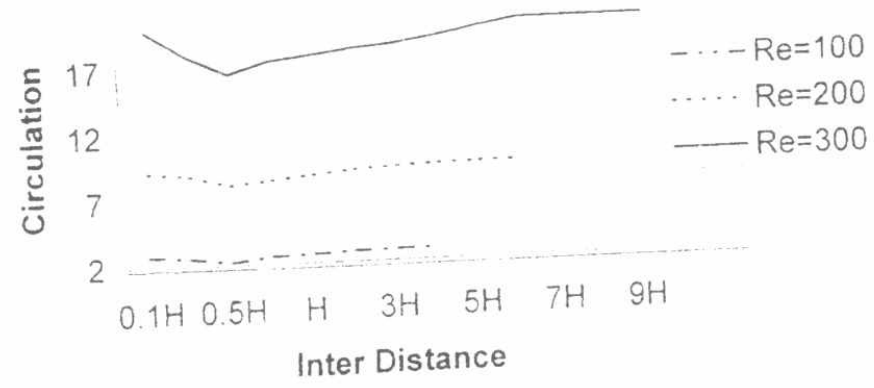

Fig. 10 The Behavior of the circulation at different inter distances between the two orifices (Results for $\mathrm{D} / \mathrm{H}=0.5$ ) 


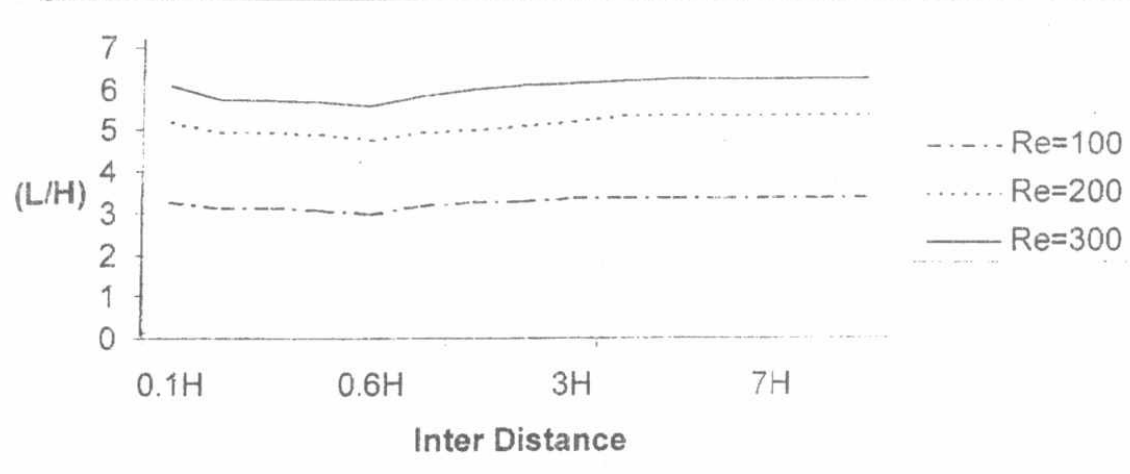

Fig. // The Behavior of the Reattachment length to the Duct height ratio at different inter distances between the two orifices

(Results for $\mathrm{D} / \mathrm{H}=0.4$ )

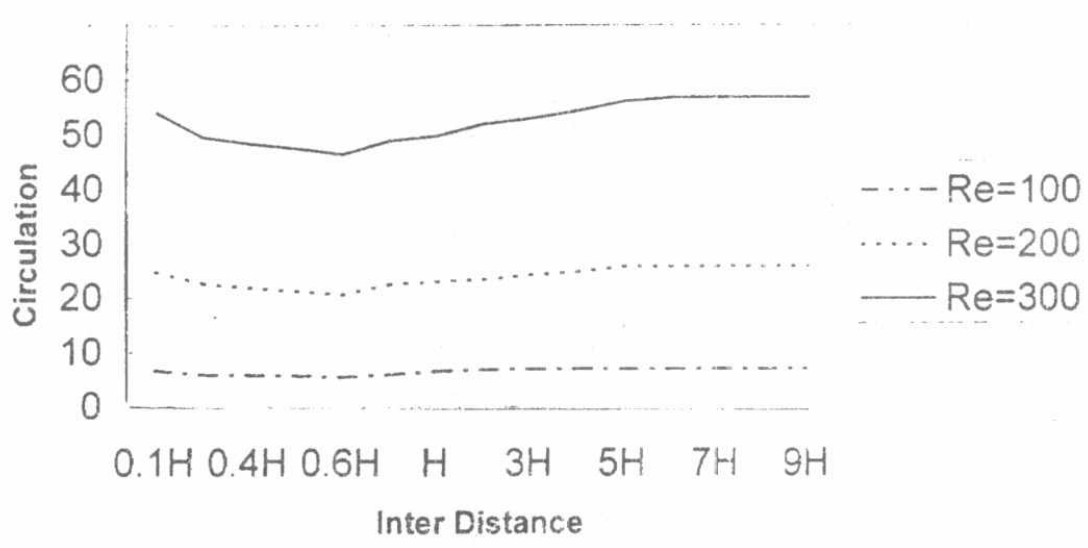

Fig. 12 The Behavior of the circulation at different inter distances between the two orifices (Results for $\mathrm{D} / \mathrm{H}=0.4$ ) 


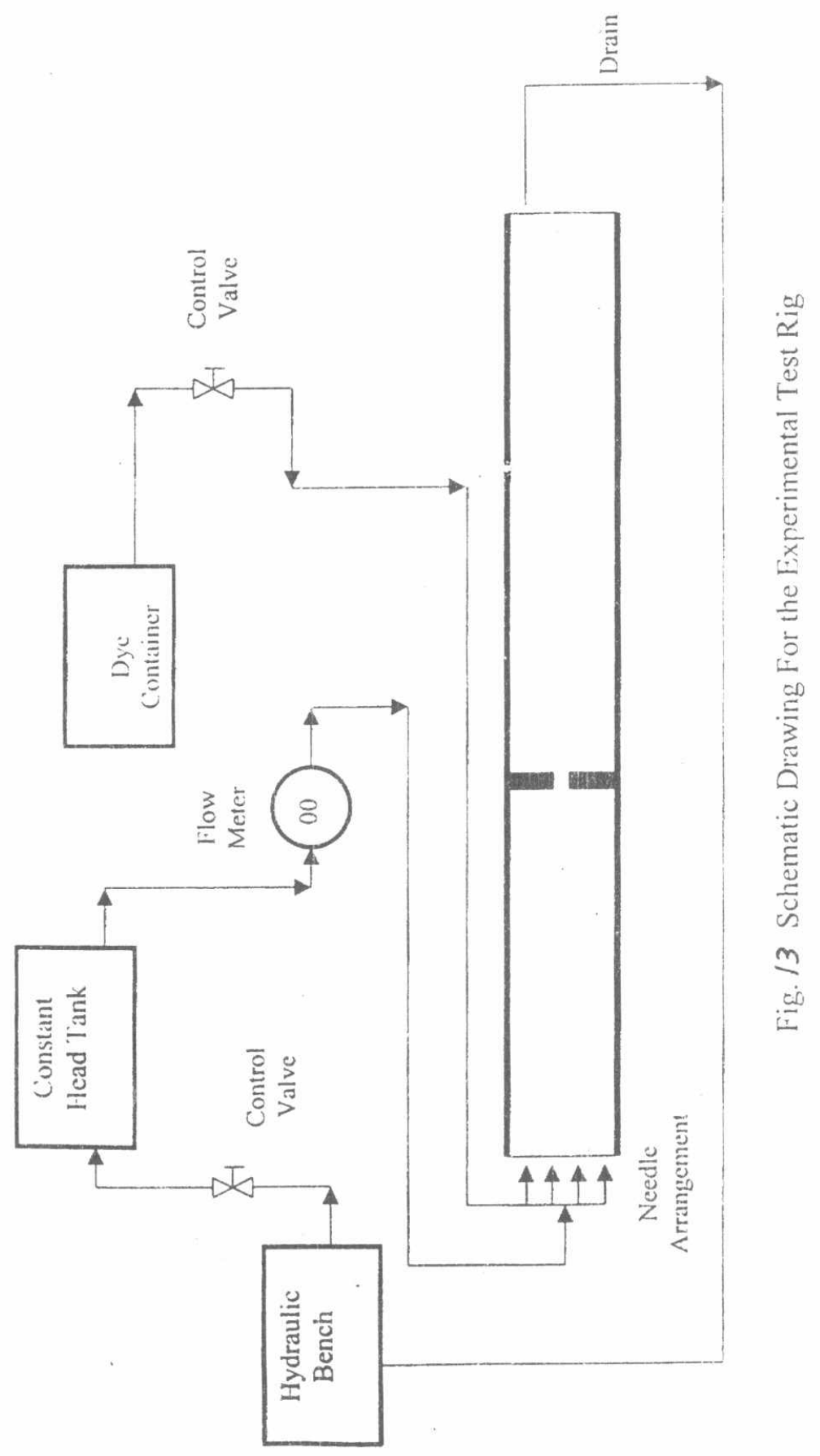




\section{Experimental Investigation:}

Different methods have been developed for flow visualization. Filaments of dye, small solid particles, globules of an immiscible liquid or minute hydrogen bubbles produced by electrolysis may be used to visualize the fluid flow. In the present work, the flow lines are visualized by the dye injection method.

The experiment was carried out in a closed loop arrangement. The test rig is shown in Fig. 13

The flow rate of water supply is adjusted to $0.25 \mathrm{~V} / \mathrm{s}$ from the pump of the hydraulic bench to the test section through a constant head tank. The chosen dye is Magnesium powder diluted in water to the ratio of 1:10.

The duct test section is fixed at $10 \mathrm{~cm}$ between the two parallel plates and $5 \mathrm{~cm}$ to the opening of the orifice; the thickness of plate sheet is $6 \mathrm{~mm}$.

\section{Experimental Results:}

The results are obtained for the range of Reynolds number from 50 to 200 and for the orifice to duct height ratios of 0.3 to 0.7

The experimental results are aimed to visualize the streamlines far from the boundary region because of the complexibilty of the dye flow near the boundaries due to no-slip condition; the flow fields are visualized for significant streamlines and compared with the results of the theoretical model.

All the plotted data are photographed by a single shoot camera at suitable position and lighting.

\section{For Single Orifice:}

The obtained results have been plotted for different orifice height to duct height ratios from 0.3 to 0.7 and for Reynolds number from 50 to 200 . Fig. 14 shows the results for $\mathrm{D} / \mathrm{H}$ from 0.3 to 0.7 .

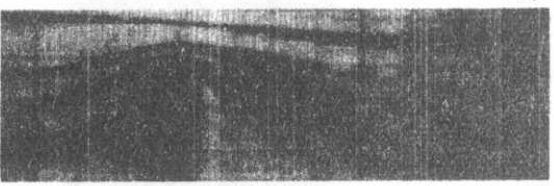

Stream lines at $\mathrm{Re}=50$

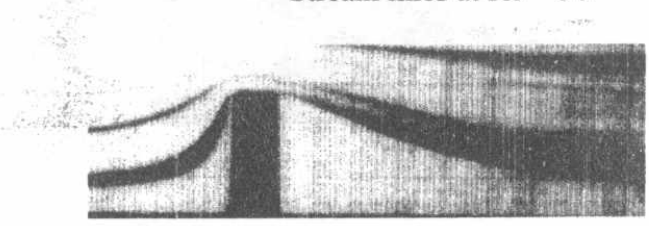

Stream lines at $\mathrm{Re}=100$ 


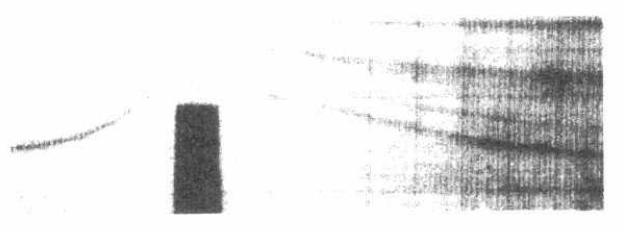

Stream lines at $\operatorname{Re}=200$

Fig. 14 Streamlines for different Reynolds numbers $(\mathrm{D} / \mathrm{H}=0.3)$

\section{For Double Orifices}

The obtained results plotted here are classified for an inter distance $0.5 \mathrm{H}$ and $\mathrm{H}$ between the two orifices the orifice height to duct height ratio is 0.5 , for the Reynolds number of 50,100 and 200.

Fig. 15 shows the results for inter distance of $\mathrm{H}$ and $0.5 \mathrm{H}$.

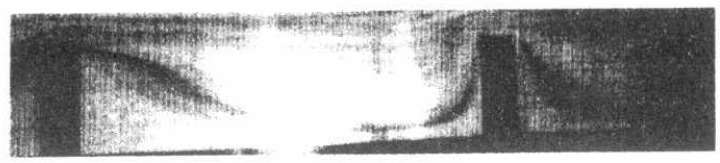

Stream lines at $\mathrm{Re}=50$

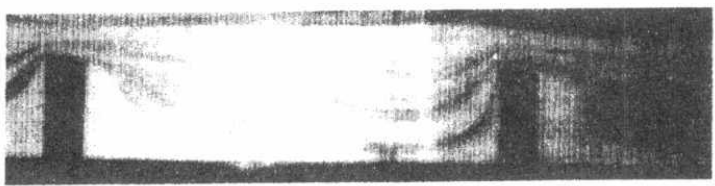

Stream lines at $\operatorname{Re}=100$

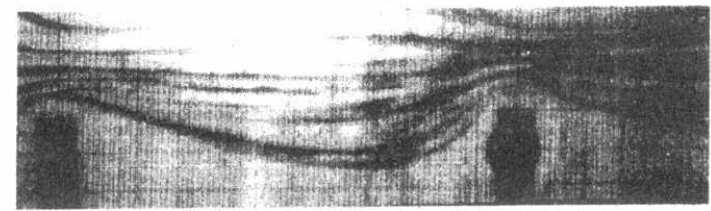

Stream lines at $R e=200$

Fig. 15 Streamlines for different Reynolds number (Inter distance $\mathrm{H}, \mathrm{D} / \mathrm{H}=0.5$ )

\section{Analysis of the Results:}

Although the flow visualization technique is a quantitative method, it could be used for comparison with the theoretical model. Measuring of the reattachment length in 
the experimental investigations has sorne difficulty because of the needie position w.r.t. the boundary which could be a disadvantage to the visualization technique near the boundary. Therefore, measuring the reattachment length could be done by taking the nearest needle to the wall and measuring its reattachment length to its original streamline. Comparing this value with the identical distance from the corresponding model.

The verification was carried out for the single orifice theoretical model with the experimental one only because the two orifices experimental results was faced with a problems of the table width and the large side wall used to make the flow uniform again.

Validating the code for the single orifice with the experimental one give a good impression about the upgrading of the used code for the two orifices and its results.

The obtained results from flow visualization through a single orifice in the duct, which has same orifice to duct height ratio and the same Reynolds number as in the theoretical model, are shown in the following plots:
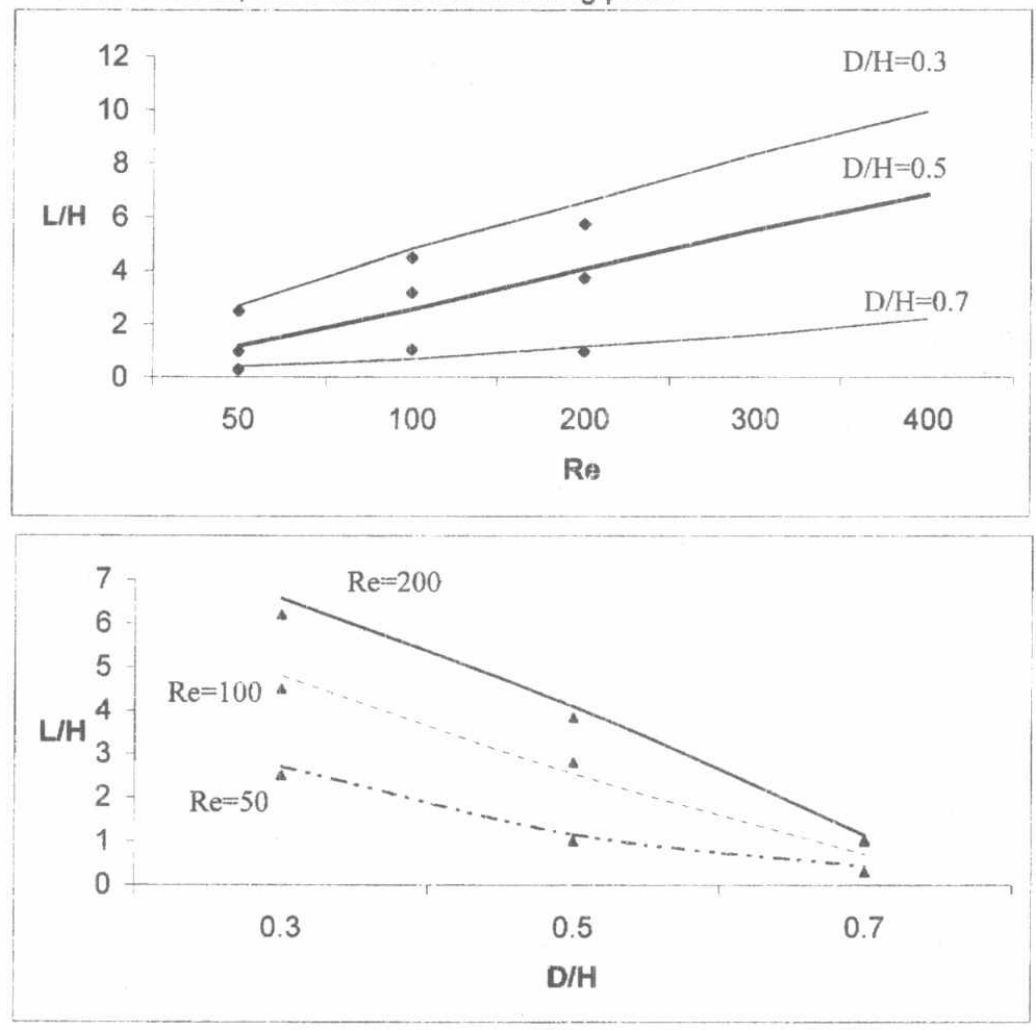

Fig. 16 Theoretical and Experimental Results 
Fig. 16 shows the experimental and theoretical results at Reynolds numbers from 50 to 200 and $\mathrm{D} / \mathrm{H}$ from 0.3 to 0.7 .

From the above results, it could be noticed that the experimental results are satisfactory matched with the theoretical model.

The results showed linear relation for $\mathrm{L} / \mathrm{H}$ as well as $\mathrm{D} / \mathrm{H}$ at different Reynolds numbers.

\section{Conclusion:}

This work presents a theoretical and experimental study for the behavior of laminar incompressible flow field through single and double orifices in series in a rectangular duct. A theoretical model based on the main governing equations of the flow (Poisson's Equation and Vorticity Transport Equation) has been written in CLanguage to determine the flow pattern through the orifice. The streamlines, the vorticity profiles, as well as the velocity distribution are obtained.

Solution has been carried for flow patterns through a single orifice and double orifices in a rectangular duct at different orifice height to duct height ratios and at different Reynolds numbers. The flow pattern through the orifice in a rectangular duct has been obtained photographically by using dye injection technique to identify the pattern after the orifice, the experimental and theoretical results showed a satisfactory agreement.

For flow through double orifices in series, in a rectangular duct in the range of $50<$ $\mathrm{Re}<400$ at $\mathrm{D} / \mathrm{H}=0.5$ and inter-distance between orifices varies from $0.1 \mathrm{H}$ to $7 \mathrm{H}$, the following are found:

1. The reattachment length of the flow downstream the second orifice changes with the inter-distance and the orifice height to duct height ratio. It has a minimum value when the inter-distance value equal to one minus the ratio of the orifice height to duct height ratio which leads to a cavity of equals sides (a square cavity) at which the minimum vortex size is generated downstream the second orifice.

2. The circulation also has minimum value at the inter distance and the orifice height to duct height ratio has a value equal to one minus the ratio of the orifice height to the duct height ratio which leads to a cavity of equals sides (a square covity) at which the minimum vortex size is generated downstream the second orifice.

\section{References:}

1. Donald Rockwell, "Flow Induced Oscillations ", Course, Department of Mechanical Engineering and Mechanics, Lehigh University, 1983.

2. Sahin B and Akilli H, "Finite Element Solution of Laminar Flow Through Square-Edged Orifice with a Variable Thickness", International Journal of Computational Fluid Dynamics", Vol. 9: (1), pp 85-88, 1997.

3. Tienfuan Kerh, J. J. Lee and L. C. Wellford, "Transient Fluid Structure Interaction In a Control valve ", Journal of Fluids Engineering, Vol. 119, pp 354 $-359,1997$.

4. Ahmed F. Ghonem and James A. Sethian, "The Effect of Reynolds Number on The Structure of Recirculating Flow", AIAA Journal, vol. 25, No. 1, 1987. 
5. D. Badekas and D. D. Knight, "Eddy Correlation For Laminar Axisymmetric Sudden Expansion Flows", Journal of Fluids Engineering, Vol. 114, pp 119120,1992

6. K. C. Chang, W. D. Hsieh and C. S. Chen, "A Modified Low Reynoldis Number Turbulence Model Applicable To Recirculating Flow In Pipe Expansion", Journal of Fluids Engineering, Vol. 117,pp 417-423, 1995.

7. Robert R. and Chia Chi Yao, "A Numerical Study of Vortex Shedding From A Square Cylinder With Ground Effect", Journal of Fluids Engineering, Vol. 119, pp 512-517, 1997.

8. Ahmed M.Salah M.Sc. Thesis, "Noise Control of Fluid Power System", 2000, Cairo.

9. Roache P. J., Computational Fluid Dynamics, Hermosa Publishers, 1976

10. Wirz H. J. and Smoldern J. J., "Numerical Methods in Fluid Dynamics", McGraw Hill, 1978.

11. Robert D. Blevins, "Applied Fluid Dynamics Handbook ", Van Nostrandhold Company, 1984.

12.A.H.Lotfy, I.Saleh, H.Abdallah and A.M.Salah, "Numerical Investigation of Flow Reattachment downstream an orifice in a Rectangular Duct", Conference of AMME, M.T.C., 2000. 